MATHEMATICS OF COMPUTATION

Volume 70, Number 234, Pages 533-553

S 0025-5718(00)01188-1

Article electronically published on March 3, 2000

\title{
FINITE VOLUME RELAXATION SCHEMES FOR MULTIDIMENSIONAL CONSERVATION LAWS
}

\author{
THEODOROS KATSAOUNIS AND CHARALAMBOS MAKRIDAKIS
}

\begin{abstract}
We consider finite volume relaxation schemes for multidimensional scalar conservation laws. These schemes are constructed by appropriate discretization of a relaxation system and it is shown to converge to the entropy solution of the conservation law with a rate of $h^{1 / 4}$ in $L^{\infty}\left([0, T], L_{\text {loc }}^{1}\left(\mathbb{R}^{d}\right)\right)$.
\end{abstract}

\section{INTRODUCTION}

In this paper we consider a class of finite volume schemes approximating the scalar multidimensional conservation law, whose construction is motivated by discretizing the relaxation system

$$
\begin{aligned}
& \partial_{t} w^{\varepsilon}+\operatorname{div} A w^{\varepsilon}=\frac{1}{\varepsilon} \sum_{i=1}^{d} G_{i}\left(w^{\varepsilon}, z_{i}^{\varepsilon}\right), x \in \mathbb{R}^{d}, \\
& \partial_{t} z_{i}^{\varepsilon}+\operatorname{div} B_{i} z_{i}^{\varepsilon}=\frac{1}{\varepsilon} G_{i}\left(w^{\varepsilon}, z_{i}^{\varepsilon}\right), i=1, \ldots, d, x \in \mathbb{R}^{d},
\end{aligned}
$$

in variables $(w, Z)$ with $Z=\left(z_{1}, \ldots, z_{d}\right)$. The constant vectors $A, B_{i}, i=1, \ldots, d$ and the smooth functions $G_{i}: \mathbb{R} \times \mathbb{R} \rightarrow \mathbb{R}$ satisfy certain structural assumptions, cf. Section 2. The system (1.1-1.2) is considered with initial data $w^{\varepsilon}(x, 0)=$ $w_{0}^{\varepsilon}(x), Z^{\varepsilon}(x, 0)=Z_{0}^{\varepsilon}(x), x \in \mathbb{R}^{d}$. Contractive relaxation systems of the form (1.11.2) were introduced and analyzed in Katsoulakis and Tzavaras KT1, and it was shown under certain assumptions that as $\varepsilon \rightarrow 0$ their solution is associated to the unique entropy solution of the conservation law,

$$
\partial_{t} u+\operatorname{div} F(u)=0, \quad x \in \mathbb{R}^{d}, t>0, \quad u(x, 0)=u_{0}(x) \in L^{1}\left(\mathbb{R}^{d}\right) \cap L^{\infty}\left(\mathbb{R}^{d}\right) .
$$

Here, for a given conservation law (1.3), we appropriately select $A, B_{i}, i=$ $1, \ldots, d$ and the functions $G_{i}$, and we discretize $(1.1-1.2)$ by semidisctere and fully discrete finite volume schemes. The approximations emanating from these schemes are shown to converge to the entropy solution of (1.3) with a rate of $h^{1 / 4}$ in $L^{\infty}\left([0, T], L_{\mathrm{loc}}^{1}\left(\mathbb{R}^{d}\right)\right)$.

Received by the editor October 31, 1997 and, in revised form, September 23, 1998, November 20, 1998, and March 9, 1999.

2000 Mathematics Subject Classification. Primary 65M12, 65M15; Secondary 65L06.

(C)2000 American Mathematical Society 


\section{Preliminaries - Relaxation SChemes}

We assume that for a given conservation law (1.3) we select the vectors $A, B_{i}, i=$ $1, \ldots, d$, and the functions $G_{i}$ such that,

$$
\begin{aligned}
& G_{i}\left(\cdot, z_{i}\right) \text { is strictly decreasing in } w \text { for fixed } z_{i}, \\
& G_{i}(w, \cdot) \text { is strictly decreasing in } z_{i} \text { for fixed } w,
\end{aligned}
$$

and that there exist functions $h_{i}: \mathbb{R} \rightarrow \mathbb{R}$,

$$
\begin{aligned}
& h_{i} \text { strictly decreasing, } h_{i}(0)=0, \quad \lim _{w \rightarrow \pm \infty} h_{i}(w)=\mp \infty, \\
& G_{i}\left(w, h_{i}(w)\right)=0, G_{i}(0,0)=0, \quad w \in \mathbb{R} .
\end{aligned}
$$

Given $\mathcal{R}^{a, b}=[a, b] \times \prod_{i=1}^{d}\left[h_{i}(b), h_{i}(a)\right]$, there exists a $\sigma=\sigma(a, b)>0$ such that

$$
\left|G_{i}\left(w, z_{i}\right)\right| \geq \sigma\left|h_{i}(w)-z_{i}\right| \quad \text { for }(w, Z) \in \mathcal{R}^{a, b},
$$

and finally, if $F$ is the flux of the conservation law (1.3), $h_{i}$ should satisfy

$$
F(\eta)=A(v)-\sum_{i=1}^{d} B_{i}\left(h_{i}(v)\right), \quad \text { if } \eta=v-\sum_{i=1}^{d} h_{i}(v), \quad v \in \mathbb{R} .
$$

Note that as a consequence of (A.1-A.3) there hold

$$
\begin{aligned}
& \left(h_{i}(w)-z_{i}\right) G_{i}\left(w, z_{i}\right)>0, \\
& \left|G_{i}\left(w, z_{i}\right)\right| \leq \sigma^{\prime}\left|h_{i}(w)-z_{i}\right| \quad \text { for }(w, Z) \in \mathcal{R}^{a, b} \text {, where } \sigma^{\prime}=\sigma^{\prime}(a, b)>0 .
\end{aligned}
$$

Lemma 4.1 of [KT1 shows that it is indeed possible to construct such functions, e.g., when $A=\left(\omega_{1} V_{1}, \ldots, \omega_{d} V_{d}\right), V_{i}>0, \omega_{i}>0, B_{i}=\left(0, \ldots,-V_{i}, \ldots, 0\right)$, and $G_{i}\left(w, z_{i}\right)=h_{i}(w)-z_{i}$, and $V_{i}, \omega_{i}$ are chosen to satisfy certain subcharacteristic conditions, cf. [KT1, [CLL] and [JX]. In this case and for $d=1$, the relaxation system (1.1) is equivalent to the one proposed by Jin and Xin [JX] and analyzed by Natalini [N1]. The convergence properties of (1.1) for $d \geq 1$ were investigated in [KT1]. In [N2] an alternative relaxation system was proposed and analyzed.

Assumptions (A.2) and (A.4) provide a (formal) reasoning on the relationship of (1.1-1.2) and (1.3). Indeed (1.1-1.2) imply that

$$
\partial_{t}\left(w^{\varepsilon}-\sum_{i=1}^{d} z_{i}^{\varepsilon}\right)+\operatorname{div}\left(A w^{\varepsilon}-\sum_{i=1}^{d} B_{i} z_{i}^{\varepsilon}\right)=0 .
$$

As $\varepsilon \rightarrow 0$ we expect that the local equilibrium, $z_{i}=h_{i}(w), i=1, \ldots, d$, will be enforced and therefore, in view of (A.4), the limiting dynamics of the relaxation system will be described by the weak solutions of (1.3), cf. [KT1. For small $\varepsilon$, $w^{\varepsilon}-\sum_{i=1}^{d} z_{i}^{\varepsilon}$ will provide an approximation to the solution $u$ of (1.3). Based on this observation one can construct approximating schemes to (1.3) by discretizing the relaxation system. The corresponding schemes are then called relaxation schemes.

Finite difference relaxation schemes were presented in a systematic way by Jin and Xin [JX]. Finite difference relaxation schemes based on the system (1.1) were proposed and analyzed in $[\mathrm{KKM}]$. It was shown that these schemes converge to the entropy solution of the multidimensional conservation law with a rate of $h^{1 / 2}$ in $L^{\infty}\left([0, T], L^{1}\left(\mathbb{R}^{d}\right)\right)$. Error estimates of difference schemes to relaxation models arising in chromatography were proved in [SCTW], ShTW]. The convergence of finite volume schemes approximating the entropy solution of (1.3) was analyzed, 
e.g., in $[\mathrm{CCL},[\mathrm{KR}, \mathrm{V}]$. In a recent paper Rohde $[\mathbf{R}]$, using an appropriate extension of DiPerna's theory, has proved convergence of finite volume schemes to weakly coupled hyperbolic systems.

Space discretization. Let $\mathcal{T}_{h}$ be a decomposition of $\mathbb{R}^{d}$ into nonoverlapping, nonempty and open polyhedra such that $\bigcup_{K \in \mathcal{T}_{h}} \bar{K}=\mathbb{R}^{d}$. The set of faces of $K$ is denoted by $\partial K$ and, on each face $e$ on $K, \nu_{e, K} \in \mathbb{R}^{d}$ represents the outward unit normal to the face $e$. $\Gamma_{h}$ will denote the set of all faces of the decomposition $\mathcal{T}_{h}$. Given a face $e$ of $K, K_{e}$ denotes the unique polyhedron that shares the face $e$ with $K$. The volume of $K$ is denoted by $|K|$ and the $(d-1)$-measure of $e$ by $|e|$. Let $h_{K}$ be the diameter of the polyhedron $K$ and let $h=\sup _{K \in \mathcal{T}_{h}} h_{K}<1$. We shall assume that our decomposition is regular, i.e., if $\rho_{K}$ is the diameter of the largest ball $B, B \subset K$,

$$
h_{K} \leq \gamma \rho_{K}, \quad K \in \mathcal{T}_{h}
$$

with a constant $\gamma$ independent of $h$. In particular this implies that if $e$ is a face of $K$, then $|e|$ and $h_{K}$ are comparable. We define the finite volume scheme approximating $(1.1),(1.2)$ as follows. We seek a piecewise constant function $\left(w_{h}, Z_{h}\right),\left.w_{h}\right|_{K}=w_{K}$, $Z_{h}=\left(z_{1, h}, \ldots, z_{d, h}\right),\left.z_{i, h}\right|_{K}=z_{i, K}$, such that

$$
\begin{aligned}
\partial_{t} w_{K}+\sum_{e \in \partial K} \frac{|e|}{|K|} g^{K}\left(w_{K}, w_{K_{e}}\right) & =\frac{1}{\varepsilon} \sum_{i=1}^{d} G_{i}\left(w_{K}, z_{i, K}\right) \\
\partial_{t} z_{i, K}+\sum_{e \in \partial K} \frac{|e|}{|K|} g_{i}^{K}\left(z_{i, K}, z_{i, K_{e}}\right) & =\frac{1}{\varepsilon} G_{i}\left(w_{K}, z_{i, K}\right), i=1, \ldots, d, K \in \mathcal{T}_{h},
\end{aligned}
$$

where $g, g_{i}: \mathbb{R} \times \mathbb{R} \rightarrow \mathbb{R}, i=1, \ldots, d$, are discrete monotone fluxes. For initial approximations we take $w_{K}(0)=\frac{1}{|K|} \int_{K} w^{0} d x$ and $z_{i, K}(0)=\frac{1}{|K|} \int_{K} z_{i}^{0} d x$. Although $g^{K}, g_{i}^{K}$ correspond to linear fluxes, it is convenient in the analysis to list their properties as in the general (nonlinear) case. We explicitly use, when it is needed, the linearity, cf. (2.6). The discrete fluxes are assumed to satisfy:

$$
g^{K}(u, v)=-g^{K_{e}}(v, u), g_{i}^{K}(u, v)=-g_{i}^{K_{e}}(v, u) \quad \text { Conservation Property, }
$$

$$
g^{K}(u, u)=A(u) \cdot \nu_{e, K}, g_{i}^{K}(u, u)=B_{i}(u) \cdot \nu_{e, K} \quad \text { Consistency Property, }
$$

$$
\frac{\partial g^{K}}{\partial u}, \frac{\partial g_{i}^{K}}{\partial u} \geq 0, \frac{\partial g^{K}}{\partial v}, \frac{\partial g_{i}^{K}}{\partial v} \leq 0 \quad \text { Monotonicity Property, }
$$

$$
g^{K}(u, v), g_{i}^{K}(u, v) \text { are linear functions of } u, v \text {. }
$$

Time discretization. Let $\delta$ be the time step and $t^{n}=n \delta$. Then an appropriate fully discrete version of (2.2) follows. We seek a piecewise constant function $\left(w_{h, \delta}, Z_{h, \delta}\right)$, $\left.w_{h, \delta}\right|_{K \times\left[t^{n}, t^{n+1}\right)}=w_{K}^{n}, \quad Z_{h, \delta}=\left(z_{1, h, \delta}, \ldots, z_{d, h, \delta}\right),\left.z_{i, h, \delta}\right|_{K \times\left[t^{n}, t^{n+1}\right)}=z_{i, K}^{n}$, 
such that,

$$
\begin{aligned}
& w_{K}^{n+1}=w_{K}^{n}-\frac{\delta}{|K|} \sum_{e \in \partial K}|e| g^{K}\left(w_{K}^{n}, w_{K_{e}}^{n}\right)+\frac{\delta}{\varepsilon} \sum_{i=1}^{d} G_{i}\left(w_{K}^{n+1}, z_{i, K}^{n+1}\right), \\
& z_{i, K}^{n+1}=z_{i, K}^{n}-\frac{\delta}{|K|} \sum_{e \in \partial K}|e| g_{i}^{K}\left(z_{i, K}^{n}, z_{i, K_{e}}^{n}\right)+\frac{\delta}{\varepsilon} G_{i}\left(w_{K}^{n+1}, z_{i, K}^{n+1}\right), \quad i=1, \ldots, d,
\end{aligned}
$$

with initial approximations $w_{K}^{0}=\frac{1}{|K|} \int_{K} w_{0}(x) d x$, and $z_{i, K}^{0}=\frac{1}{|K|} \int_{K} z_{i 0}(x) d x, i=$ $1, \ldots, d$.

The stability and convergence properties of the semidiscrete scheme are investigated in the next sections. We prove that under standard assumptions on the initial data, for any $R>0, T>0$, there is a constant $C=C(R, T)$ such that

$$
\left\|u(\cdot, t)-U_{h}(\cdot, t)\right\|_{L^{1}(B(0, R))} \leq C h^{1 / 4}, \quad t \leq T,
$$

where $U_{h}=w_{h}-\sum_{i=1}^{d} h_{i}\left(w_{h}\right)$, cf. Theorem 4.1. Here $B(0, R)$ is the ball with center 0 and radius $R$. In the case of fully discrete approximations a similar estimate holds true, provided that appropriate CFL conditions are valid, cf. Theorem 5.1.

A main advantage of relaxation schemes, is the simplicity of their construction coming from the fact that the principal part of (1.1-1.2) is linear, and therefore there is no need to solve local Riemann problems. Thus high order and adaptive schemes can be easily formulated. Issues related to the numerical implementation and the performance of finite volume relaxation schemes are addressed in [KZ].

Error estimates of order $O\left(h^{1 / 4}\right)$ for finite volume approximations to (1.3) were previously obtained in [CL], [V], and for finite elements in CG1. For finite difference approximations the order of convergence $O\left(h^{1 / 2}\right)$ was established, e.g., in $[\mathrm{Kz}],[\mathrm{S}]$. The main reason for the reduced order of convergence in the finite volume case is the lack of BV bounds for the approximate schemes in unstructured meshes. To compensate for this, an estimate for the discrete gradients in $L^{2}$ was proved in [CCL], $\mathrm{V}$ ], which led to the $O\left(h^{1 / 4}\right)$ estimate. In the case of relaxation schemes considered here we are able to prove an analogous bound, cf. Lemma 3.3. In addition for the relaxation schemes, again due to the lack of BV bounds, an estimate for the distance from the equilibrium in $L^{2}$ turns out to be crucial, cf. Lemma 3.4. It is to be noted that Lemma 3.3 provides a rigorous proof of the dissipative character of relaxation schemes (in the finite volume as well as in the finite difference setting), compare with [JX.

Our analysis is based on an approximation lemma for deriving error estimates for numerical approximations to conservation law (1.3), cf. Lemma 4.1. This is a result obtained in [KKM] and extends a result of Bouchut and Perthame [BP to the case of numerical schemes. The use of this lemma in the (complicated) case of finite volume approximations considered in this paper avoids much of the technical work needed if one applies the original approach of doubling the variables, $\mathrm{Kr}$, $[\mathrm{Kz}$, as in [CCL], $\mathrm{V}]$. Indeed, the analysis in [CCL], [V] is considerably simplified if one uses Lemma 4.1 along the lines of the analysis presented in Section 4, cf. also GM]. As noted first in [CG2, the classical approach of Kuznetsov is an "a posteriori" approach. This can be seen directly in the framework considered in this paper, simply by observing that the E-terms in the bound (4.5) depend only on the approximate solution $u_{h}$. Indeed, by applying a more refined analysis, explicit 
a posteriori error bounds suitable for adaptive mesh refinement based on Lemma 4.1 are proposed in GM for finite difference and finite volume approximations to (1.3), cf. also [CGa].

An alternative "a priori" approach for deriving error estimates, which does not rely on the regularity properties of the schemes, was proposed and extensively analyzed in CG2, CG3] for finite difference and in [CGY] for finite volume schemes. To carry out the program proposed in [CG1] one has to show an appropriate "discrete" stability for the scheme considered, a task considerably more complicated than the "continuous" stability used in the proof of Lemma 4.1. Cockburn, Gremaud and Yang in CGY] were able to prove $h^{1 / 2}$ estimates by using this approach for a special class of monotone finite volume schemes in symmetric (or nearly symmetric) non-Cartesian meshes, cf. [CGY, Sections 2.a, b] for explicit assumptions.

The paper is organized as follows. In Section 3 we prove the necessary stability properties for schemes. We then use these properties in Section 4 to prove convergence. In particular the relaxation schemes satisfy a basic comparison principle (Lemma 3.1) which then implies the $L^{1}$ contraction property (Lemma 3.2), the fact that $\mathcal{R}^{a, b}$ is a positively invariant region for the schemes and as consequence that the approximations are uniformly bounded in $L^{\infty}$ (Lemma 3.2), and the discrete entropy inequalities (3.8). Using the invariance of $\mathcal{R}^{a, b}$ we are then able to show the weak dissipation estimates (Lemma 3.3) and the estimate for the distance from equilibrium (Lemma 3.4) mentioned above. In the convergence proof of Section 4 we first use the discrete entropy to prove the basic error inequality (4.15) which then allows us to apply Lemma 4.1. To estimate then the $E$-terms of (4.5) we use Lemmata 3.3 and 3.4.

\section{Stability estimates}

We first prove a comparison principle which implies several useful properties of the scheme. We start by introducing some notation. For $a, b \in \mathbb{R}$ we set $a \vee b=$ $\max \{a, b\}$ and $a \wedge b=\min \{a, b\}$. Further, for a given function $f$ we denote by $f^{+}, f^{-}$the positive and negative parts of $f$, respectively, and $\chi_{f>0}$ stands for the characteristic function of the set $\{(x, t): f(x, t)>0\}$, that is $\chi_{f>0}=1$ if $f>0$ and zero if $f \leq 0$.

Lemma 3.1. Assume that $G_{i}(\cdot, \cdot), i=1, \ldots, d$, satisfy assumptions (A.1-A.3). Let $\left(w_{h}, Z_{h}\right)$ and $\left(\bar{w}_{h}, \bar{Z}_{h}\right)$ be two solutions of $(2.2)$ that vanish outside a ball $B_{M}$ of radius $M$. Then we have

$$
\begin{aligned}
\partial_{t}\left\{\left(w_{K}-\bar{w}_{K}\right)^{+}+\right. & \left.\sum_{i=1}^{d}\left(z_{i, K}-\bar{z}_{i, K}\right)^{-}\right\} \\
+ & \sum_{e \in \partial K} \frac{|e|}{|K|} \chi_{w_{K}-\bar{w}_{K}>0}\left\{g^{K}\left(w_{K} \vee \bar{w}_{K}, w_{K_{e}} \vee \bar{w}_{K_{e}}\right)\right. \\
& \left.\quad-g^{K}\left(w_{K} \wedge \bar{w}_{K}, w_{K_{e}} \wedge \bar{w}_{K_{e}}\right)\right\} \\
+ & \sum_{i=1}^{d} \sum_{e \in \partial K} \frac{|e|}{|K|} \chi_{z_{i, K}-\bar{z}_{i, K}<0}\left\{g_{i}^{K}\left(z_{i, K} \vee \bar{z}_{i, K}, z_{i, K_{e}} \vee \bar{z}_{i, K_{e}}\right)\right. \\
& \left.-g_{i}^{K}\left(z_{i, K} \wedge \bar{z}_{i, K}, z_{i, K_{e}} \wedge \bar{z}_{i, K_{e}}\right)\right\} \leq 0
\end{aligned}
$$


Proof. Let $\left(w_{h}, Z_{h}\right)$ and $\left(\bar{w}_{h}, \bar{Z}_{h}\right)$ be two solutions of (2.2). Then we have

$$
\begin{aligned}
& \partial_{t}\left(w_{K}-\bar{w}_{K}\right)+\sum_{e \in \partial K} \frac{|e|}{|K|}\left\{g^{K}\left(w_{K}, w_{K_{e}}\right)-g^{K}\left(\bar{w}_{K}, \bar{w}_{K_{e}}\right)\right\} \\
& =\frac{1}{\varepsilon} \sum_{i=1}^{d}\left\{G_{i}\left(w_{K}, z_{i, K}\right)-G_{i}\left(\bar{w}_{K}, \bar{z}_{i, K}\right)\right\} \\
& \partial_{t}\left(z_{i, K}-\bar{z}_{i, K}\right)+\sum_{e \in \partial K} \frac{|e|}{|K|}\left\{g_{i}^{K}\left(z_{i, K}, z_{i, K_{e}}\right)-g_{i}^{K}\left(\bar{z}_{i, K}, \bar{z}_{i, K_{e}}\right)\right\} \\
& =\frac{1}{\varepsilon}\left\{G_{i}\left(w_{K}, z_{i, K}\right)-G_{i}\left(\bar{w}_{K}, \bar{z}_{i, K}\right)\right\} .
\end{aligned}
$$

Using the fact that $f^{+}=\chi_{f>0} f, f^{-}=-\chi_{f<0} f$ multiplying the first equation by $\chi_{w_{K}-\bar{w}_{K}>0}$, and the second by $-\chi_{z_{i, K}-\bar{z}_{i, K}<0}$ summing over $i$ and adding the resulting equations, we get by using the monotonicity assumptions on $G_{i}$

$$
\begin{aligned}
\partial_{t}\left\{\left(w_{K}-\bar{w}_{K}\right)^{+}\right. & \left.+\sum_{i=1}^{d}\left(z_{i, K}-\bar{z}_{i, K}\right)^{-}\right\} \\
& +\sum_{e \in \partial K} \frac{|e|}{|K|} \chi_{w_{K}-\bar{w}_{K}>0}\left[g^{K}\left(w_{K}, w_{K_{e}}\right)-g^{K}\left(\bar{w}_{K}, \bar{w}_{K_{e}}\right)\right] \\
& -\sum_{i=1}^{d} \sum_{e \in \partial K} \frac{|e|}{|K|} \chi_{z_{i, K}-\bar{z}_{i, K}<0}\left[g_{i}^{K}\left(z_{i, K}, z_{i, K_{e}}\right)-g_{i}^{K}\left(\bar{z}_{i, K}, \bar{z}_{i, K_{e}}\right)\right] \leq 0 .
\end{aligned}
$$

Let

$$
\mathcal{T}_{w}=-\chi_{w_{K}-\bar{w}_{K}>0}\left[g^{K}\left(w_{K}, w_{K_{e}}\right)-g^{K}\left(\bar{w}_{K}, \bar{w}_{K_{e}}\right)\right]
$$

and

$$
\mathcal{T}_{z}=\chi_{z_{i, K}-\bar{z}_{i, K}<0}\left[g_{i}^{K}\left(z_{i, K}, z_{i, K_{e}}\right)-g_{i}^{K}\left(\bar{z}_{i, K}, \bar{z}_{i, K_{e}}\right)\right] .
$$

Then we have the following.

(a) For $w_{K}-\bar{w}_{K}>0$, we have that $w_{K}=w_{K} \vee \bar{w}_{K}$ and $\bar{w}_{K}=w_{K} \wedge \bar{w}_{K}$; otherwise $\mathcal{T}_{w}=0$. Then, using (2.5) we have

$$
\mathcal{T}_{w} \leq-\chi_{w_{K}-\bar{w}_{K}>0}\left\{g^{K}\left(w_{K} \vee \bar{w}_{K}, w_{K_{e}} \vee \bar{w}_{K_{e}}\right)-g^{K}\left(w_{K} \wedge \bar{w}_{K}, w_{K_{e}} \wedge \bar{w}_{K_{e}}\right)\right\}
$$

(b) Similarly, for $z_{i, K}-\bar{z}_{i, K}<0$ (otherwise $\mathcal{T}_{z}=0$ ) we have that $z_{i, K}=z_{i, K} \wedge \bar{z}_{i, K}$ and $\bar{z}_{i, K}=z_{i, K} \vee \bar{z}_{i, K}$. Now, (2.5) implies

$\mathcal{T}_{z} \leq-\chi_{z_{i, K}-\bar{z}_{i, K}<0}\left\{g_{i}^{K}\left(z_{i, K} \vee \bar{z}_{i, K}, z_{i, K_{e}} \vee \bar{z}_{i, K_{e}}\right)-g_{i}^{K}\left(z_{i, K} \wedge \bar{z}_{i, K}, z_{i, K_{e}} \wedge \bar{z}_{i, K_{e}}\right)\right\}$

Therefore, (3.2), (3.3) and (3.4) yield (3.1).

Next, we show that the scheme is $L^{1}$ contractive and bounded in $L^{\infty}$. 
Lemma 3.2. Under the assumptions of Lemma 3.1 we have

$$
\begin{aligned}
& \left\|w_{h}(t)-\bar{w}_{h}(t)\right\|_{L^{1}}+\sum_{i=1}^{d}\left\|z_{i, h}(t)-\bar{z}_{i, h}(x, t)\right\|_{L^{1}} \\
& \leq\left\|w_{h}(\tau)-\bar{w}_{h}(\tau)\right\|_{L^{1}}+\sum_{i=1}^{d}\left\|z_{i, h}(\tau)-\bar{z}_{i, h}(\tau)\right\|_{L^{1}}, \quad 0 \leq \tau<t .
\end{aligned}
$$

(ii) If, for some $a<b$, we have $a \leq w_{K}(0) \leq b, h_{i}(b) \leq z_{i, K}(0) \leq h_{i}(a), i=$ $1, \ldots, d, K \in \mathcal{T}_{h}$, then

$$
a \leq w_{K}(t) \leq b, h_{i}(b) \leq z_{i, K}(t) \leq h_{i}(a), K \in \mathcal{T}_{h}, i=1, \ldots, d,
$$

i.e., the region $\mathcal{R}^{a, b}=[a, b] \times \prod_{i=1}^{d}\left[h_{i}(b), h_{i}(a)\right]$ is positively invariant.

Proof. (i) Relation (3.1) implies

$$
\begin{aligned}
& \partial_{t}\left\{\left|w_{K}-\bar{w}_{K}\right|+\sum_{i=1}^{d}\left|z_{i, K}-\bar{z}_{i, K}\right|\right\} \\
& \quad+\sum_{e \in \partial K} \frac{|e|}{|K|} g^{K}\left(\left|w_{K}-\bar{w}_{K}\right|,\left|w_{K_{e}}-\bar{w}_{K_{e}}\right|\right) \\
& \quad+\sum_{i=1}^{d} \sum_{e \in \partial K} \frac{|e|}{|K|} g_{i}^{K}\left(\left|z_{i, K}-\bar{z}_{i, K}\right|,\left|z_{i, K_{e}}-\bar{z}_{i, K_{e}}\right|\right) \leq 0 .
\end{aligned}
$$

Multiplying by $|K|$ and then summing w.r. to $K \in \mathcal{T}_{h}$, we get (i) by noticing that in each edge of our partition,

$$
g^{K}\left(\left|w_{K}-\bar{w}_{K}\right|,\left|w_{K_{e}}-\bar{w}_{K_{e}}\right|\right)+g^{K_{e}}\left(\left|w_{K_{e}}-\bar{w}_{K_{e}}\right|,\left|w_{K}-\bar{w}_{K}\right|\right)=0,
$$

and a similar relation for the $z_{i, K}$ terms.

For the proof of (ii), we first observe that

$$
\begin{gathered}
\chi_{w_{K}-\bar{w}_{K}>0} g^{K}\left(w_{K}-\bar{w}_{K}, w_{K_{e}}-\bar{w}_{K_{e}}\right) \\
\geq g^{K}\left(\left(w_{K}-\bar{w}_{K}\right)^{+},\left(w_{K_{e}}-\bar{w}_{K_{e}}\right)^{+}\right), \\
-\chi_{z_{i, K}-\bar{z}_{i, K}<0} g_{i}^{K}\left(z_{i, K}-\bar{z}_{i, K}, z_{i, K_{e}}-\bar{z}_{i, K_{e}}\right) \\
\geq-g_{i}^{K}\left(\left(z_{i, K}-\bar{z}_{i, K}\right)^{-},\left(z_{i, K_{e}}-\bar{z}_{i, K_{e}}\right)^{-}\right) .
\end{gathered}
$$

Indeed, by the monotonicity properties of $g^{K}$, if $\chi_{w_{K}-\bar{w}_{K}>0}=1$,

$$
\begin{aligned}
\chi_{w_{K}-\bar{w}_{K}>0} g^{K}\left(w_{K}-\bar{w}_{K}, w_{K_{e}}-\bar{w}_{K_{e}}\right) & =g^{K}\left(\left(w_{K}-\bar{w}_{K}\right)^{+},\left(w_{K_{e}}-\bar{w}_{K_{e}}\right)\right) \\
& \geq g^{K}\left(\left(w_{K}-\bar{w}_{K}\right)^{+},\left(w_{K_{e}}-\bar{w}_{K_{e}}\right)^{+}\right),
\end{aligned}
$$

in the case where $\chi_{w_{K}-\bar{w}_{K}>0}=0$ it suffices to show $g^{K}\left(0,\left(w_{K_{e}}-\bar{w}_{K_{e}}\right)^{+}\right) \leq 0$. But this is a consequence of $(2.3),(2.4)$ and the fact that $\left(w_{K_{e}}-\bar{w}_{K_{e}}\right)^{+} \geq 0$. The corresponding relation for $z_{i, K}$ is proved similarly.

Using now these relations, the fact that $g^{K}\left(\left(w_{K}-\bar{w}_{K}\right)^{+},\left(w_{K_{e}}-\bar{w}_{K_{e}}\right)^{+}\right)+$ $g^{K_{e}}\left(\left(w_{K_{e}}-\bar{w}_{K_{e}}\right)^{+},\left(w_{K}-\bar{w}_{K}\right)^{+}\right)=0$, and the corresponding relation for $z_{i, K}$ we 
obtain in view of (3.2),

$$
\begin{aligned}
& \sum_{K \in \mathcal{T}_{h}}|K|\left[\left(w_{K}(t)-\bar{w}_{K}(t)\right)^{+}+\sum_{i}\left(z_{i, K}(t)-\bar{z}_{i, K}(t)\right)^{-}\right] \\
& \leq \sum_{K \in \mathcal{T}_{h}}|K|\left[\left(w_{K}(0)-\bar{w}_{K}(0)\right)^{+}+\sum_{i}\left(z_{i, K}(0)-\bar{z}_{i, K}(0)\right)^{-}\right] .
\end{aligned}
$$

Then, (ii) follows by noticing that $\bar{w}_{K}=b, \bar{z}_{i, K}=h_{i}(b)$ is a solution of the semidiscrete scheme, since $\sum_{e \in \partial K}|e| g^{K}(a, a)=0$ and $\sum_{e \in \partial K}|e| g_{i}^{K}\left(h_{i}(b), h_{i}(b)\right)=0$.

Discrete entropy inequality. Lemma 3.1 implies a discrete entropy inequality. Indeed (3.1) is still valid if we interchange + and - . For any $\xi \in \mathbb{R}$, we let $\bar{w}_{K}=$ $\xi, \bar{z}_{i, K}=h_{i}(\xi), i=1, \ldots, d$, and setting, for $u, v \in \mathbb{R}$

$$
\begin{gathered}
D_{\xi}^{K}(u, v)=g^{K}(u \vee \xi, v \vee \xi)-g^{K}(u \wedge \xi, v \wedge \xi)=g^{K}(|u-\xi|,|v-\xi|), \\
\begin{aligned}
D_{\xi}^{i, K}(u, v) & =g_{i}^{K}\left(u \vee h_{i}(\xi), v \vee h_{i}(\xi)\right)-g_{i}^{K}\left(u \wedge h_{i}(\xi), v \wedge h_{i}(\xi)\right) \\
& =g_{i}^{K}\left(\left|u-h_{i}(\xi)\right|,\left|v-h_{i}(\xi)\right|\right)
\end{aligned}
\end{gathered}
$$

we get after summation using (3.1) the following discrete entropy inequality

$$
\begin{aligned}
\partial_{t}\left\{\left|w_{K}-\xi\right|\right. & \left.+\sum_{i=1}^{d}\left|z_{i, K}-h_{i}(\xi)\right|\right\} \\
& +\sum_{e \in \partial K} \frac{|e|}{|K|}\left\{D_{\xi}^{K}\left(w_{K}, w_{K_{e}}\right)+\sum_{i=1}^{d} D_{\xi}^{i, K}\left(z_{i, K}, z_{i, K_{e}}\right)\right\} \leq 0 .
\end{aligned}
$$

Remark 3.1. Notice that for $D_{\xi}^{K}$ we have, for $u \in \mathbb{R}$,

$$
D_{\xi}^{K}(u, u)=|u-\xi| A \cdot \nu_{e, K} \text { and } D_{\xi}^{i, K}(u, u)=\left|u-h_{i}(\xi)\right| B_{i} \cdot \nu_{e, K} .
$$

Dissipation estimate. The next lemma provides an estimate for the distance from the equilibrium $z_{i}=h_{i}(w)$ for our approximating scheme and a weak dissipation estimate for $w_{K}$ and $z_{i, K}$. A stronger estimate for the distance from the equilibrium is proved in Lemma 3.4. This result compensates for the lack of BV estimates for our schemes (compare with [CCL, [V] in the proof of the convergence result in Section 4 . We need some more notation: Let $h_{i}^{-1}$ denote the inverse of $h_{i}$, and

$$
\Psi_{i}(z)=-\int_{0}^{z} h_{i}^{-1}(\xi) d \xi
$$

cf., [KT1]. The functions $\Psi_{i}, i=1, \ldots, d$, are positive and strictly convex according to our assumptions on $h_{i}$, cf. Section 2. In particular (A.2) implies that there exists $\mu=\mu(a, b)>0$ such that

$$
\Psi_{i}^{\prime \prime}(z) \geq \mu>0, \quad z \in\left[h_{i}(b), h_{i}(a)\right] .
$$

Our assumptions on the fluxes imply that

$$
\begin{aligned}
& g^{K}(u, v)=\frac{A \cdot \nu_{e, K}}{2}(u+v)+a_{\nu_{e, K}}(u-v), \\
& g_{i}^{K}(u, v)=\frac{B_{i} \cdot \nu_{e, K}}{2}(u+v)+b_{\nu_{e, K}}^{i}(u-v),
\end{aligned}
$$

where $a_{e}:=a_{\nu_{e, K}}=a_{\nu_{e, K_{e}}} \geq 0$ and $b_{e}^{i}:=b_{\nu_{e, K}}^{i}=b_{\nu_{e} K_{e}}^{i} \geq 0$. (2.5) implies $\frac{1}{2}\left|A \cdot \nu_{e, K}\right| \leq a_{e}$ and $\frac{1}{2}\left|B_{i} \cdot \nu_{e, K}\right| \leq b_{e}^{i}$. 
Remark 3.2. Most of the well-known monotone fluxes are reduced to the linear case, e.g., for $g^{K}(u, v)$ to

$$
g^{K}(u, v)=\frac{A \cdot \nu_{e, K}}{2}(u+v)+\frac{\left|A \cdot \nu_{e, K}\right|}{2}(u-v) .
$$

We now have

Lemma 3.3. Assume that the initial conditions satisfy $\left(w_{h}^{0}, Z_{h}^{0}\right) \in \mathcal{R}^{a, b}$ for some $a, b \in \mathbb{R}$. Then if $\sigma=\sigma(a, b)$ and $\mu=\mu(a, b)$ are the constants of (A.3) and (3.9), respectively, there holds

$$
\begin{aligned}
\frac{\sigma}{\varepsilon} \int_{0}^{t} \sum_{K \in \mathcal{T}_{h}}|K| \sum_{i=1}^{d}\left(h_{i}\left(w_{K}\right)-z_{i, K}\right)^{2} & \\
& +\int_{0}^{t} \sum_{e \in \Gamma_{h}}|e|\left\{a_{e}\left(w_{K}-w_{K_{e}}\right)^{2}+\mu \sum_{i=1}^{d} b_{e}^{i}\left(z_{i, K}-z_{i, K_{e}}\right)^{2}\right\} \\
\leq & \sum_{K \in \mathcal{T}_{h}}|K|\left\{\frac{1}{2}\left(w_{K}^{0}\right)^{2}+\sum_{i=1}^{d} \Psi_{i}\left(z_{i, K}^{0}\right)\right\} \leq C
\end{aligned}
$$

where $a_{e}, b_{e}^{i}$ are as defined in (3.10).

Proof. First we notice that (2.4) implies

$$
\sum_{e \in \partial K}|e| g^{K}\left(w_{K}, w_{K}\right)=0, \sum_{e \in \partial K}|e| g_{i}^{K}\left(z_{i, K}, z_{i, K}\right)=0 .
$$

We then multiply (2.2a) by $w_{K}$ and $(2.2 \mathrm{~b})$ by $h_{i}^{-1}\left(z_{i, K}\right)$, sum over $i$ and subtract the resulting equations. Next, if we multiply by $|K|$ and sum, we finally obtain

$$
\begin{aligned}
\sum_{K \in \mathcal{T}_{h}}|K| \partial_{t} & \left\{\frac{1}{2} w_{K}^{2}+\sum_{i=1}^{d} \Psi_{i}\left(z_{i, K}\right)\right\}+\sigma \frac{1}{\varepsilon} \sum_{K \in \mathcal{T}_{h}}|K| \sum_{i=1}^{d}\left(h_{i}\left(w_{K}\right)-z_{i, K}\right)^{2} \\
& +\sum_{e \in \Gamma_{h}}|e|\left\{w_{K} g^{K}\left(w_{K}, w_{K_{e}}\right)+w_{K_{e}} g^{K_{e}}\left(w_{K_{e}}, w_{K}\right)\right\} \\
& -\sum_{i=1}^{d} \sum_{e \in \Gamma_{h}}|e|\left\{h_{i}^{-1}\left(z_{i, K}\right)\left[g_{i}^{K}\left(z_{i, K}, z_{i, K_{e}}\right)-g_{i}^{K}\left(z_{i, K}, z_{i, K}\right)\right]\right. \\
& \left.+h_{i}^{-1}\left(z_{i, K_{e}}\right)\left[g_{i}^{K_{e}}\left(z_{i, K_{e}}, z_{i, K}\right)-g_{i}^{K_{e}}\left(z_{i, K_{e}}, z_{i, K_{e}}\right)\right]\right\} \leq 0
\end{aligned}
$$

where we have used that $-\left(w-h_{i}^{-1}(z)\right) G_{i}\left(w, z_{i}\right) \geq \sigma\left(h_{i}(w)-z_{i}\right)^{2}$, cf. (A.1-A.3), $\left(\right.$ A. $\left.3^{\prime}\right)$. We will first estimate the terms corresponding to $w$-fluxes. Using (3.10) we get

$$
\sum_{e \in \Gamma_{h}}|e|\left\{w_{K} g^{K}\left(w_{K}, w_{K_{e}}\right)+w_{K_{e}} g^{K_{e}}\left(w_{K_{e}}, w_{K}\right)\right\}=\sum_{e \in \Gamma_{h}}|e| a_{e}\left(w_{K}-w_{K_{e}}\right)^{2},
$$


since

$$
\begin{aligned}
& \sum_{e \in \Gamma_{h}}|e| \frac{1}{2} A \cdot \nu_{e, K}\left(w_{K}^{2}-w_{K_{e}}^{2}\right)=\sum_{e \in \Gamma_{h}}|e|\left\{\frac{1}{2} A \cdot \nu_{e, K} w_{K}^{2}+\frac{1}{2} A \cdot \nu_{e, K_{e}} w_{K_{e}}^{2}\right\} \\
&=\sum_{K \in \mathcal{T}_{h}} \sum_{e \in \partial K}|e| \frac{1}{2} A \cdot \nu_{e, K} w_{K}^{2}=0 .
\end{aligned}
$$

For the $z$ fluxes of (3.11), using (3.10) for $g_{i}^{K}\left(z_{i, K}, z_{i, K_{e}}\right)$, we first write

$$
\begin{array}{r}
-h_{i}^{-1}\left(z_{i, K}\right)\left[g_{i}^{K}\left(z_{i, K}, z_{i, K_{e}}\right)-g_{i}^{K}\left(z_{i, K}, z_{i, K}\right)\right] \\
=\Psi^{\prime}\left(z_{i, K}\right)\left[\frac{1}{2} B_{i} \cdot \nu_{e, K}-b_{e}^{i}\right]\left(z_{i, K_{e}}-z_{i, K}\right),
\end{array}
$$

where, as before, $b_{e}^{i}=b_{\nu_{e, K}}^{i}=b_{\nu_{e, K}}^{i}$. By (2.5) $\frac{1}{2} B_{i} \cdot \nu_{e, K}-b_{e}^{i} \leq 0$, and hence using Taylor's formula and (3.9), $\Psi_{i}^{\prime}\left(c_{1}\right)\left(c_{2}-c_{1}\right) \leq \Psi\left(c_{2}\right)-\Psi\left(c_{1}\right)-\frac{\mu}{2}\left(c_{1}-c_{2}\right)^{2}$, we get

$$
\begin{gathered}
-h_{i}^{-1}\left(z_{i, K}\right)\left[g_{i}^{K}\left(z_{i, K}, z_{i, K_{e}}\right)-g_{i}^{K}\left(z_{i, K}, z_{i, K}\right)\right] \\
\geq\left[\frac{1}{2} B_{i} \cdot \nu_{e, K}-b_{e}^{i}\right]\left(\Psi_{i}\left(z_{i, K_{e}}\right)-\Psi_{i}\left(z_{i, K}\right)\right) \\
+\frac{1}{2} \mu\left[b_{e}^{i}-\frac{1}{2} B_{i} \cdot \nu_{e, K}\right]\left(z_{i, K}-z_{i, K_{e}}\right)^{2} .
\end{gathered}
$$

Similarly,

$$
\begin{gathered}
-h_{i}^{-1}\left(z_{i, K_{e}}\right)\left[g_{i}^{K_{e}}\left(z_{i, K_{e}}, z_{i, K}\right)-g_{i}^{K_{e}}\left(z_{i, K_{e}}, z_{i, K_{e}}\right)\right] \\
\geq\left[\frac{1}{2} B_{i} \cdot \nu_{e, K_{e}}-b_{e}^{i}\right]\left(\Psi_{i}\left(z_{i, K}\right)-\Psi_{i}\left(z_{i, K_{e}}\right)\right) \\
+\frac{1}{2} \mu\left[b_{e}^{i}-\frac{1}{2} B_{i} \cdot \nu_{e, K_{e}}\right]\left(z_{i, K}-z_{i, K_{e}}\right)^{2} .
\end{gathered}
$$

But then

$$
\begin{aligned}
\sum_{e \in \Gamma_{h}}|e|\left\{\left[\frac{1}{2} B_{i} \cdot \nu_{e, K}-b_{e}^{i}\right]\left(\Psi_{i}\left(z_{i, K_{e}}\right)-\Psi_{i}\left(z_{i, K}\right)\right)\right. & \left.+\left[\frac{1}{2} B_{i} \cdot \nu_{e, K_{e}}-b_{e}^{i}\right]\left(\Psi_{i}\left(z_{i, K}\right)-\Psi_{i}\left(z_{i, K_{e}}\right)\right)\right\} \\
= & \sum_{e \in \Gamma_{h}}|e| B_{i} \cdot \nu_{e, K}\left(\Psi_{i}\left(z_{i, K_{e}}\right)-\Psi_{i}\left(z_{i, K}\right)\right) \\
= & -\sum_{e \in \Gamma_{h}}|e|\left\{B_{i} \cdot \nu_{e, K} \Psi_{i}\left(z_{i, K}\right)+B_{i} \cdot \nu_{e, K_{e}} \Psi_{i}\left(z_{i, K_{e}}\right)\right\} \\
= & -\sum_{K \in \mathcal{T}_{h}} \sum_{e \in \partial K}|e| B_{i} \cdot \nu_{e, K} \Psi_{i}\left(z_{i, K}\right)=0 .
\end{aligned}
$$

Therefore,

$$
\begin{aligned}
&-\sum_{e \in \Gamma_{h}}|e|\{ h_{i}^{-1}\left(z_{i, K}\right)\left[g_{i}^{K}\left(z_{i, K}, z_{i, K_{e}}\right)-g_{i}^{K}\left(z_{i, K}, z_{i, K}\right)\right] \\
&\left.\quad+h_{i}^{-1}\left(z_{i, K_{e}}\right)\left[g_{i}^{K_{e}}\left(z_{i, K_{e}}, z_{i, K}\right)-g_{i}^{K_{e}}\left(z_{i, K_{e}}, z_{i, K_{e}}\right)\right]\right\} \\
& \geq \sum_{e \in \Gamma_{h}}|e| \mu b_{e}^{i}\left(z_{i, K}-z_{i, K_{e}}\right)^{2} .
\end{aligned}
$$


In view of these estimates (3.11) implies

$$
\begin{aligned}
\sum_{K \in \mathcal{T}_{h}}|K| \partial_{t} & \left\{\frac{1}{2} w_{K}^{2}+\sum_{i=1}^{d} \Psi_{i}\left(z_{i, K}\right)\right\}+\sigma \frac{1}{\varepsilon} \sum_{K \in \mathcal{T}_{h}}|K| \sum_{i=1}^{d}\left(h_{i}\left(w_{K}\right)-z_{i, K}\right)^{2} \\
& +\sum_{e \in \Gamma_{h}}|e| a_{e}\left(w_{K}-w_{K_{e}}\right)^{2}+\sum_{i=1}^{d} \sum_{e \in \Gamma_{h}}|e| \mu b_{e}^{i}\left(z_{i, K}-z_{i, K_{e}}\right)^{2} \leq 0
\end{aligned}
$$

and the proof is complete.

Distance from equilibrium. Next, we estimate

$$
\sup _{t} \sum_{K \in \mathcal{T}_{h}}|K| \sum_{i}\left|G_{i}\left(w_{K}(t), z_{i, K}(t)\right)\right|^{2} .
$$

Lemma 3.4. Let $\left(w_{h}, Z_{h}\right)$ be a solution of the semidiscrete scheme emanating from data with finite total variation and lying in an (invariant) region $\mathcal{R}^{a, b}$. Assume further that

$$
\sum_{K \in \mathcal{T}_{h}}|K| \sum_{i=1}^{d}\left|G_{i}\left(w_{K}(0), z_{i, K}(0)\right)\right|^{2} \leq C \varepsilon .
$$

Then, for any $1>\eta>0$ there exists a constant $C_{\eta}=C(\eta, a, b)$ such that

$$
\sup _{t} \sum_{K \in \mathcal{T}_{h}}|K| \sum_{i}\left|G_{i}\left(w_{K}(t), z_{i, K}(t)\right)\right|^{2} \leq C_{\eta} \varepsilon^{\prime}, \quad \text { with } \varepsilon^{\prime}=\varepsilon^{1-\eta} .
$$

Proof. Using the definition of the scheme, we have

$$
\begin{aligned}
\partial_{t} G_{i}\left(w_{K}(t), z_{i, K}(t)\right)= & \frac{\partial G_{i}}{\partial w}\left\{-\sum_{e \in \partial K} \frac{|e|}{|K|} g^{K}\left(w_{K}, w_{K_{e}}\right)+\frac{1}{\varepsilon} \sum_{j=1}^{d} G_{j}\left(w_{K}(t), z_{j, K}(t)\right)\right\} \\
& +\frac{\partial G_{i}}{\partial z}\left\{-\sum_{e \in \partial K} \frac{|e|}{|K|} g_{i}^{K}\left(z_{i, K}, z_{i, K_{e}}\right)+\frac{1}{\varepsilon} G_{i}\left(w_{K}(t), z_{i, K}(t)\right)\right\} .
\end{aligned}
$$

Multiplying by $G_{i}\left(w_{K}, z_{i, K}\right)=G_{i}$ and adding, we obtain

$$
\begin{aligned}
& \frac{1}{2} \partial_{t} \sum_{i}\left|G_{i}\left(w_{K}, z_{i, K}\right)\right|^{2}+\frac{1}{\varepsilon} \sum_{i}\left(-\frac{\partial G_{i}}{\partial z}\right)\left|G_{i}\left(w_{K}, z_{i, K}\right)\right|^{2} \\
& =\frac{1}{\varepsilon} \sum_{i=1}^{d} \frac{\partial G_{i}}{\partial w} G_{i}\left(w_{K}, z_{i, K}\right) \sum_{j=1}^{d} G_{j}\left(w_{K}, z_{j, K}\right) \\
& +\sum_{i=1}^{d} G_{i}\left(\frac{\partial G_{i}}{\partial w}\left[-\sum_{e \in \partial K} \frac{|e|}{|K|}\left(g^{K}\left(w_{K}, w_{K_{e}}\right)-g^{K}\left(w_{K}, w_{K}\right)\right)\right]\right. \\
& \left.\quad+\frac{\partial G_{i}}{\partial z}\left[-\sum_{e \in \partial K} \frac{|e|}{|K|}\left(g_{i}^{K}\left(z_{i, K}, z_{i, K_{e}}\right)-g_{i}^{K}\left(z_{i, K}, z_{i, K}\right)\right)\right]\right) .
\end{aligned}
$$

Observe now that (A.2) implies that $-\frac{\partial G_{i}}{\partial z}>c_{1}=c_{1}(a, b)>0$ in $\mathcal{R}^{a, b}$. Also, $\left|g^{K}\left(w_{K}, w_{K_{e}}\right)-g^{K}\left(w_{K}, w_{K}\right)\right| \leq a_{e}\left|w_{K}-w_{K_{e}}\right|$. Therefore, if $\varepsilon \leq C h_{K}, K \in \mathcal{T}_{h}$, there exists a constant $c_{0}=c_{0}(a, b)>0$, such that

$$
\partial_{t} \sum_{K \in \mathcal{T}_{h}}|K| \sum_{i}\left|G_{i}\left(w_{K}, z_{i, K}\right)\right|^{2}+\frac{c_{0}}{\varepsilon} \sum_{K \in \mathcal{T}_{h}}|K| \sum_{i}\left|G_{i}\left(w_{K}, z_{i, K}\right)\right|^{2} \leq C \mathcal{A}
$$


where

$$
\begin{aligned}
\mathcal{A}= & \sigma \frac{1}{\varepsilon} \sum_{K \in \mathcal{T}_{h}}|K| \sum_{i=1}^{d}\left(h_{i}\left(w_{K}\right)-z_{i, K}\right)^{2} \\
& +\sum_{e \in \Gamma_{h}}|e| a_{e}\left(w_{K}-w_{K_{e}}\right)^{2}+\sum_{i=1}^{d} \sum_{e \in \Gamma_{h}}|e| \mu b_{e}^{i}\left(z_{i, K}-z_{i, K_{e}}\right)^{2},
\end{aligned}
$$

and Lemma 3.3 implies that, for any $t>0, \int_{0}^{t} \mathcal{A}(s) d s \leq C$. By (3.14) and our assumption on the initial data, we have

$$
\begin{aligned}
& \sum_{K \in \mathcal{T}_{h}}|K| \sum_{i}\left|G_{i}\left(w_{K}(t), z_{i, K}(t)\right)\right|^{2} \\
& \quad \leq e^{-\frac{c_{0}}{\varepsilon} t} \sum_{K \in \mathcal{T}_{h}}|K| \sum_{i}\left|G_{i}\left(w_{K}(0), z_{i, K}(0)\right)\right|^{2}+C \int_{0}^{t} e^{-\frac{c_{0}}{\varepsilon}(t-s)} \mathcal{A}(s) d s \\
& \quad \leq C \varepsilon+C \int_{0}^{t} e^{-\frac{c_{0}}{\varepsilon}(t-s)} \mathcal{A}(s) d s .
\end{aligned}
$$

Let $1>\eta>0$ be an arbitrarily small number and $\varepsilon^{\prime}=\varepsilon^{1-\eta}$. The proof of the lemma will be complete if we show

$$
\int_{0}^{t} e^{-\frac{c_{0}}{\varepsilon}(t-s)} \mathcal{A}(s) d s \leq C_{\eta} \varepsilon^{\prime} .
$$

Since $\frac{1}{\varepsilon} e^{-\frac{c_{0}}{\varepsilon^{\eta}}}$ is bounded for $\varepsilon \rightarrow 0$, we have, in view of Lemma 3.3,

$$
\int_{0}^{t-\varepsilon^{\prime}} e^{-\frac{c_{0}}{\varepsilon}(t-s)} \mathcal{A}(s) d s \leq \varepsilon \int_{0}^{t-\varepsilon^{\prime}} \frac{1}{\varepsilon} e^{-\frac{c_{0}}{\varepsilon \eta}} \mathcal{A}(s) d s \leq c \varepsilon \int_{0}^{t-\varepsilon^{\prime}} \mathcal{A}(s) d s \leq c \varepsilon .
$$

On the other hand, for $N_{\varepsilon}=\left[\varepsilon^{-\eta}\right]+1$,

$$
\int_{t-\varepsilon^{\prime}}^{t} e^{-\frac{c_{0}}{\varepsilon}(t-s)} \mathcal{A}(s) d s \leq \int_{t-N_{\varepsilon} \varepsilon}^{t} \mathcal{A}(s) d s \leq \sum_{m=1}^{N_{\varepsilon}} \int_{t-m \varepsilon}^{t-(m-1) \varepsilon} \mathcal{A}(s) d s .
$$

Then using (3.12), we obtain

$$
\begin{aligned}
& \int_{t-m \varepsilon}^{t-(m-1) \varepsilon} \mathcal{A}(s) d s \\
& \leq C \sum_{K \in \mathcal{T}_{h}}|K|\left\{\left|w_{K}^{2}(t-m \varepsilon)-w_{K}^{2}(t-(m-1) \varepsilon)\right|\right. \\
& \left.\quad+\sum_{i=1}^{d}\left|\Psi_{i}\left(z_{i, K}(t-m \varepsilon)\right)-\Psi_{i}\left(z_{i, K}(t-(m-1) \varepsilon)\right)\right|\right\} \\
& \leq C^{\prime}(a, b) \sum_{K \in \mathcal{T}_{h}}|K|\left\{\left|w_{K}(t-m \varepsilon)-w_{K}(t-(m-1) \varepsilon)\right|\right. \\
& \left.\quad+\sum_{i=1}^{d}\left|z_{i, K}(t-m \varepsilon)-z_{i, K}(t-(m-1) \varepsilon)\right|\right\} \\
& \leq C^{\prime}(a, b) \sum_{K \in \mathcal{T}_{h}}|K|\left\{\left|w_{K}(\varepsilon)-w_{K}(0)\right|+\sum_{i=1}^{d}\left|z_{i, K}(\varepsilon)-z_{i, K}(0)\right|\right\}
\end{aligned}
$$


Here we also used that $\left(w_{h}, Z_{h}\right) \in \mathcal{R}^{a, b}$, and the $L^{1}$ contraction property (Lemma $3.2(\mathrm{i}))$ for $\left(\bar{w}_{h}(\cdot), \bar{Z}_{h}(\cdot)\right)=\left(w_{h}(\cdot+\varepsilon), Z_{h}(\cdot+\varepsilon)\right)$. Let us assume that

$$
\sum_{K \in \mathcal{T}_{h}}|K|\left\{\left|w_{K}(\varepsilon)-w_{K}(0)\right|+\sum_{i=1}^{d}\left|z_{i, K}(\varepsilon)-z_{i, K}(0)\right|\right\} \leq C \varepsilon .
$$

Then, (3.16), (3.17) and (3.18) imply

$$
\int_{t-\varepsilon^{\prime}}^{t} e^{-\frac{c_{0}}{\varepsilon}(t-s)} \mathcal{A}(s) d s \leq C N_{\varepsilon} \varepsilon=C\left(\left[\varepsilon^{-\eta}\right]+1\right) \varepsilon \leq C \varepsilon^{\prime},
$$

and the proof of (3.15) (and therefore of Lemma 3.4) will be complete. Hence it remains to verify (3.18). To this end let $0<\tau \leq \varepsilon$. Then, by (2.2), we see that

$$
\begin{aligned}
|K|\left\{\left|w_{K}(\tau)-w_{K}(0)\right|\right. & \left.+\sum_{i=1}^{d}\left|z_{i, K}(\tau)-z_{i, K}(0)\right|\right\} \\
\leq C \int_{0}^{\tau} \sum_{e \in \partial K}|e|\left(\left|w_{K}-w_{K_{e}}\right|\right. & +\sum_{i=1}^{d}\left|z_{i, K}-z_{i, K_{e}}\right| \\
& \left.+\frac{1}{\varepsilon} \sum_{i=1}^{d}|K|\left|G_{i}\left(w_{K}, z_{i, K}\right)\right|\right) d s .
\end{aligned}
$$

We estimate the terms of the right-hand side as follows:

$$
\begin{aligned}
\left|G_{i}\left(w_{K}(s), z_{i, K}(s)\right)\right| \leq & \left|G_{i}\left(w_{K}(0), z_{i, K}(0)\right)\right| \\
& +C(a, b)\left(\left|w_{K}(s)-w_{K}(0)\right|+\left|z_{i, K}(s)-z_{i, K}(0)\right|\right)
\end{aligned}
$$

and

$$
\left|w_{K}(s)-w_{K_{e}}(s)\right| \leq\left|w_{K}(0)-w_{K_{e}}(0)\right|+\left|w_{K}(s)-w_{K}(0)\right|+\left|w_{K_{e}}(s)-w_{K_{e}}(0)\right| .
$$

Therefore in view of the stability of the local $L^{2}$ projection in BV, cf. [C], and our assumptions on the initial data, we have upon summing over $K$ and using again the fact that $\varepsilon \leq C h_{K}, K \in \mathcal{T}_{h}$,

$$
\begin{aligned}
\sum_{K \in \mathcal{T}_{h}}|K| & \left\{\left|w_{K}(\tau)-w_{K}(0)\right|+\sum_{i=1}^{d}\left|z_{i, K}(\tau)-z_{i, K}(0)\right|\right\} \\
& \leq C \tau+\frac{1}{\varepsilon} \int_{0}^{\tau} \sum_{K \in \mathcal{T}_{h}}|K|\left\{\left|w_{K}(s)-w_{K}(0)\right|+\sum_{i=1}^{d}\left|z_{i, K}(s)-z_{i, K}(0)\right|\right\} d s .
\end{aligned}
$$

Then, since $\tau \leq \varepsilon$, Gronwall's lemma implies

$$
\sum_{K \in \mathcal{T}_{h}}|K|\left\{\left|w_{K}(\tau)-w_{K}(0)\right|+\sum_{i=1}^{d}\left|z_{i, K}(\tau)-z_{i, K}(0)\right|\right\} \leq C e^{C \frac{\tau}{\varepsilon}} \tau \leq C \varepsilon .
$$

The proof is thus complete.

\section{Convergence}

Our convergence results will be based on the following approximation lemma [KKM], which provides a compact form for deriving error estimates for numerical approximations to conservation law (1.3). Lemma 4.1 is an extension of a result of Bouchut and Perthame $[\mathrm{BP}]$, and allows the explicit treatment of terms that 
typically arise in numerical schemes. See also EG for a result providing estimates in the space-time $L^{1}$ norm.

Lemma $4.1([\mathrm{KKM}])$. Let $u_{h}, u \in L_{\mathrm{loc}}^{\infty}\left([0, \infty), L_{\mathrm{loc}}^{1}\left(\mathbb{R}^{d}\right)\right)$ be right continuous in $t$, with values in $L_{\mathrm{loc}}^{1}\left(\mathbb{R}^{d}\right)$. Assume that $u$ is the entropy solution of a given conservation law, i.e., it satisfies (1.3) and

$$
\partial_{t}|u-k|+\sum_{i=1}^{d} \partial_{x_{i}}\left[\left(F_{i}(u)-F_{i}(k)\right) \operatorname{sgn}(u-k)\right] \leq 0, \quad \text { in } \mathcal{D}^{\prime}, \text { for all } k \in \mathbb{R},
$$

with initial value $u^{0} \in B V\left(\mathbb{R}^{d}\right)$. Let $\Psi$ be a nonnegative test function, $\Psi \in$ $C_{c}^{\infty}\left((0, \infty) \times \mathbb{R}^{d}\right)$, and assume that $u_{h}$ with initial value $u_{h}^{0}$ satisfies

$$
\begin{gathered}
-\iint_{(0, \infty) \times \mathbb{R}^{d}}\left(\left|u_{h}-k\right| \partial_{t} \Psi+\operatorname{sgn}\left(u_{h}-k\right)\left[F\left(u_{h}\right)-F(k)\right] \cdot \nabla_{x} \Psi\right) d t d x \\
\leq \iint_{(0, \infty) \times \mathbb{R}^{d}}\left(\alpha_{G}\left|\partial_{t} \Psi\right|+\sum_{j} \alpha_{H}^{j}\left|\frac{\partial \Psi}{\partial x_{j}}\right|+\sum_{j} \beta_{H}^{j} B_{H}^{j}\left(\frac{\partial \Psi}{\partial x_{j}}\right)\right) d x d t \\
\text { for all } k \in \mathbb{R},
\end{gathered}
$$

where $F=\left(F_{1}, \ldots, F_{d}\right)$ and $\alpha_{G}, \alpha_{H}^{j}, \beta_{G}, \beta_{H}^{j}$, are nonnegative $k$-independent functions in $L_{\mathrm{loc}}^{1}\left([0, \infty) \times \mathbb{R}^{d}\right)$ and $\alpha_{G}, \quad \beta_{G} \in L_{\mathrm{loc}}^{\infty}\left([0, \infty), L_{\mathrm{loc}}^{1}\left(\mathbb{R}^{d}\right)\right)$.

Let $\Delta>0$ and $\mathcal{S}_{h}=\{S\}$ be a given decomposition of $[0, \infty) \times \mathbb{R}^{d}$, into elements $S$, such that

$$
\operatorname{diam}\left(S_{t}\right) \leq \Delta, \quad \text { if } \beta_{H}^{j} \text {, is not identically zero for some } j, j=1, \ldots, d,
$$

where $S_{t}=\{x:(t, x) \in S\}$. In addition, the $k$-independent operators $B_{H}^{j}$ satisfy

$$
\left|B_{H}^{j}\left(\frac{\partial \Psi}{\partial x_{j}}\right)(t, x)\right| \leq C \sup _{x^{\prime} \in S_{t}}\left|\frac{\partial \Psi}{\partial x_{j}}\left(t, x^{\prime}\right)\right|, \quad \text { for all }(t, x) \in S, 1 \leq i, j \leq d .
$$

Here $C$ is a uniform constant independent of $\Psi$ and the element decomposition $\mathcal{S}_{h}$. Then the following estimate holds. For any $T \geq 0, x_{0} \in \mathbb{R}^{d}, R>0, \nu \geq 0$, with $M=\operatorname{Lip}(F)$, we have:

$$
\begin{aligned}
& \int_{\left|x-x_{0}\right|<R}\left|u_{h}(T, x)-u(T, x)\right| d x \\
& \quad \leq \int_{B_{0}}\left|u_{h}(0, x)-u(0, x)\right| d x+\left(M \Delta^{\prime}+\Delta\right) T V\left(u^{0}\right)+C\left(E^{G}+E^{H}+\tilde{E}^{H}\right) .
\end{aligned}
$$

Here

$$
\begin{aligned}
& E^{H}=\frac{1}{\Delta} \sum_{j=1}^{d} \iint_{0 \leq t \leq T} \alpha_{x \in B_{t}} \alpha_{H}^{j}(t, x) d x d t, \\
& \tilde{E}^{H}=\frac{1}{\Delta} \sum_{j=1}^{d} \iint_{0 \leq t \leq T} \beta_{x \in B_{t}^{\Delta}}^{j}(t, x) d x d t, \\
& E^{G}=\left(1+\frac{T}{\Delta^{\prime}}+\frac{M T}{\Delta+\nu}\right) \sup _{0 \leq t \leq 2 T} \int_{B_{t}} \alpha_{G}(t, x) d x
\end{aligned}
$$

and $B_{t}=B\left(x_{0}, R+M(T-t)+\Delta+\nu\right), B_{t}^{\Delta}=B\left(x_{0}, R+M(T-t)+2 \Delta+\nu\right)$. 
Remark 4.1. The terms $B_{H}^{j}, \beta_{H}^{j}, j=1, \ldots, d$, in (4.2), (4.4) can be replaced by one term $B_{H}, \beta_{H}$. In this case (4.4) will be

$$
\left|B_{H}\left(\nabla_{x} \Psi(t, x)\right)\right| \leq C \sup _{x \in S_{t}}\left|\nabla_{x} \Psi(t, x)\right|,
$$

and $\tilde{E}^{H}$ will be modified accordingly.

We will use Lemma 4.1 to prove our convergence results. We introduce notation that will be used along with some preliminary results. In particular, for any $k \in \mathbb{R}$ we define $\xi \in \mathbb{R}$ such that

$$
k=\xi-\sum_{i=1}^{d} h_{i}(\xi)
$$

and we set

$$
U_{h}=w_{h}-\sum_{i=1}^{d} h_{i}\left(w_{h}\right) \text { i.e., } U_{K}=w_{K}-\sum_{i=1}^{d} h_{i}\left(w_{K}\right), K \in \mathcal{T}_{h} .
$$

Then $U_{K}-k=\left[w_{K}-\xi\right]-\sum_{i=1}^{d}\left[h_{i}\left(w_{K}\right)-h_{i}(\xi)\right]$, and, since we assumed that the functions $h_{i}, i=1, \ldots, d$, are decreasing, we get

$$
\left|U_{K}-k\right|=\left|w_{K}-\xi\right|+\sum_{i=1}^{d}\left|h_{i}\left(w_{K}\right)-h_{i}(\xi)\right|,
$$

i.e.,

$$
\left|U_{K}-k\right|=\left|w_{K}-\xi\right|+\sum_{i=1}^{d}\left|z_{i, K}-h_{i}(\xi)\right|+J_{K} \quad \text { with }\left|J_{K}\right| \leq \frac{1}{\sigma} \sum_{i=1}^{d}\left|G_{i}\left(w_{K}, z_{i, K}\right)\right| .
$$

In view of (A.4) we have $F\left(U_{K}\right)=A\left(w_{K}\right)-\sum_{i=1}^{d} B_{i}\left(h_{i}\left(w_{K}\right)\right)$. Hence

$$
\begin{aligned}
& {\left[F\left(U_{K}\right)-F(k)\right] \operatorname{sgn}\left(U_{K}-k\right)} \\
& \quad=\left\{\left[A\left(w_{K}\right)-A(\xi)\right]-\sum_{i=1}^{d}\left[B_{i}\left(h_{i}\left(w_{K}\right)\right)-B_{i}\left(h_{i}(\xi)\right)\right]\right\} \operatorname{sgn}\left(U_{K}-k\right) .
\end{aligned}
$$

Now for $w_{K}-\xi>0$, we have by (A.2), $h_{i}\left(w_{K}\right)-h_{i}(\xi)<0$, hence,

$$
\operatorname{sgn}\left[\left(w_{K}-\xi\right)-\sum_{i=1}^{d}\left(h_{i}\left(w_{K}\right)-h_{i}(\xi)\right)\right]>0 .
$$

So, by (4.7) we get

$$
\left[F\left(U_{K}\right)-F(k)\right] \operatorname{sgn}\left(U_{K}-k\right)=\left|w_{K}-\xi\right| A+\sum_{i=1}^{d}\left|h_{i}\left(w_{K}\right)-h_{i}(\xi)\right| B_{i} .
$$


Similarly, (4.7a) holds if $w_{K}-\xi<0$. Therefore,

$$
\begin{array}{r}
{\left[F\left(U_{K}\right)-F(k)\right] \operatorname{sgn}\left(U_{K}-k\right)=\left|w_{K}-\xi\right| A+\sum_{i=1}^{d}\left|z_{i, K}-h_{i}(\xi)\right| B_{i}+H_{K},} \\
\quad \text { with }\left|H_{K}\right| \leq \sum_{i=1}^{d}\left|h_{i}\left(w_{K}\right)-z_{i, K}\right|\left|B_{i}\right| .
\end{array}
$$

Now we are ready to prove

Theorem 4.1. Let $u$ be the entropy solution of the conservation law (1.3) with initial data $u_{0} \in B V\left(\mathbb{R}^{d}\right) \cap L^{\infty}\left(\mathbb{R}^{d}\right)$. For $U_{h}=w_{h}-\sum_{i=1}^{d} h_{i}\left(w_{h}\right)$, where $\left(w_{h}, Z_{h}\right)$ is the solution of the semidiscrete finite volume scheme (2.2), assume that the assumptions of Lemma 3.4 hold and for some $1>\eta>0, \varepsilon^{1-\eta} \leq C h$. Then, for any time $t \leq T$ and $R>0$, there exists a constant $C=(R+M T)^{d / 4} T^{1 / 2} c(a, b)$ such that the following error estimate holds

$$
\left\|u(\cdot, t)-U_{h}(\cdot, t)\right\|_{L^{1}(B(0, R))} \leq C h^{1 / 4}+\left\|u(\cdot, 0)-U_{h}(\cdot, 0)\right\|_{L^{1}} .
$$

If in addition $\left\|u_{0}-\left(w_{0}^{\varepsilon}-\sum_{i=1}^{d} h_{i}\left(w_{0}^{\varepsilon}\right)\right)\right\|_{L^{1}} \leq C \varepsilon$, then

$$
\left\|u(\cdot, t)-U_{h}(\cdot, t)\right\|_{L^{1}(B(0, R))} \leq C h^{1 / 4} .
$$

Proof. To apply Lemma 4.1 we consider a nonnegative test function $\Psi$ with compact support, $\operatorname{supp} \Psi=\Omega$. We also set

$$
V_{K}:=\left|U_{K}-k\right| \quad \text { and } \quad V_{F, K}:=\left[F\left(U_{K}\right)-F(k)\right] \operatorname{sgn}\left(U_{K}-k\right) .
$$

Then, we would like to estimate the following quantity

$$
E:=-\int \sum_{K \in \mathcal{T}_{h}} \int_{K}\left[V_{K} \Psi_{t}+V_{F, K} \cdot \nabla_{x} \Psi\right] d x d t=:-\left(E_{1}+E_{2}\right) .
$$

For the first term, we have

$$
\begin{aligned}
E_{1} & =\int \sum_{K \in \mathcal{T}_{h}} \int_{K} V_{K} \Psi_{t} d x d t=\int \sum_{K \in \mathcal{T}_{h}} V_{K} \int_{K} \Psi_{t} d x d t \\
& =\sum_{K \in \mathcal{T}_{h}} \int V_{K} \bar{\Psi}_{t}^{K} d t \quad \text { where } \quad \bar{\Psi}_{t}^{K}=\int_{K} \Psi_{t} d t .
\end{aligned}
$$

For the second term we have,

$$
\begin{aligned}
E_{2} & =\int \sum_{K \in \mathcal{T}_{h}} V_{F, K} \cdot \int_{K} \nabla_{x} \Psi d x d t=\int \sum_{K \in \mathcal{T}_{h}} \sum_{e \in \partial K} V_{F, K} \cdot \nu_{e, K} \int_{e} \Psi d s d t \\
& =\int \sum_{e \in \Gamma_{h}}\left(V_{F, K} \cdot \nu_{e, K}+V_{F, K_{e}} \cdot \nu_{e, K_{e}}\right) \bar{\Psi}^{e} d t \quad \text { where } \bar{\Psi}^{e}=\int_{e} \Psi d s .
\end{aligned}
$$

Now (4.8) and (3.7) (cf. Remark 3.1) imply

$$
V_{F, K} \cdot \nu_{e, K}=D_{\xi}^{K}\left(w_{K}, w_{K}\right)+\sum_{i=1}^{d} D_{\xi}^{i, K}\left(z_{i, K}, z_{i, K}\right)+H_{K} \cdot \nu_{e, K} .
$$


Combining (4.9), (4.10) and (4.11) we have

$$
\begin{aligned}
E= & -\sum_{K \in \mathcal{T}_{h}} \int\left\{\left|w_{K}-\xi\right|+\sum_{i=1}^{d}\left|z_{i, K}-h_{i}(\xi)\right|\right\} \bar{\Psi}_{t}^{K} d t \\
- & \sum_{e \in \Gamma_{h}} \int\left\{\left[D_{\xi}^{K}\left(w_{K}, w_{K}\right)+D_{\xi}^{K_{e}}\left(w_{K_{e}}, w_{K_{e}}\right)\right]\right. \\
& \left.+\sum_{i=1}^{d}\left[D_{\xi}^{i, K}\left(z_{i, K}, z_{i, K}\right)+D_{\xi}^{i, K_{e}}\left(z_{i, K_{e}}, z_{i, K_{e}}\right)\right]\right\} \bar{\Psi}^{e} d t \\
- & \sum_{K \in \mathcal{T}_{h}}\left(\int J_{K} \bar{\Psi}_{t}^{K} d t+\int H_{K} \cdot \int_{K} \nabla_{x} \Psi d x d t\right) .
\end{aligned}
$$

There holds $\sum_{e \in \partial K}|e| D_{\xi}^{K}\left(w_{K}, w_{K}\right)=0$ and $\sum_{e \in \partial K}|e| D_{\xi}^{i, K}\left(z_{i, K}, z_{i, K}\right)=0, i=$ $1, \ldots, d$. Thus, if we multiply the discrete entropy inequality (3.8) by $\bar{\Psi}^{K}$ and sum for all $K \in \mathcal{T}_{h}$, and get

$$
\begin{aligned}
-\sum_{K \in \mathcal{T}_{h}} \int\left\{\left|w_{K}-\xi\right|+\sum_{i=1}^{d}\left|z_{i, K}-h_{i}(\xi)\right|\right\} \bar{\Psi}_{t}^{K} d t \\
+\sum_{e \in \Gamma_{h}} \int|e|\left\{\left(\mathcal{F}_{w}^{K}+\mathcal{F}_{w}^{K_{e}}\right)+\sum_{i=1}^{d}\left(\mathcal{F}_{z_{i}}^{K}+\mathcal{F}_{z_{i}}^{K_{e}}\right)\right\} d t \leq 0,
\end{aligned}
$$

where

$$
\begin{gathered}
\mathcal{F}_{w}^{K}=\frac{1}{|K|}\left[D_{\xi}^{K}\left(w_{K}, w_{K_{e}}\right)-D_{\xi}^{K}\left(w_{K}, w_{K}\right)\right] \bar{\Psi}^{K}, \\
\mathcal{F}_{z_{i}}^{K}=\frac{1}{|K|}\left[D_{\xi}^{i, K}\left(z_{i, K}, z_{i, K_{e}}\right)-D_{\xi}^{i, K}\left(z_{i, K}, z_{i, K}\right)\right] \bar{\Psi}^{K},
\end{gathered}
$$

and $\mathcal{F}_{w}^{K_{e}}, \mathcal{F}_{z_{i}}^{K_{e}}$ are defined by the same formulas with $K$ and $K_{e}$ interchanged. In view of (4.13), we see that (4.12) implies

$$
\begin{aligned}
E \leq & -\sum_{e \in \Gamma_{h}} \int|e|\left\{\left(\mathcal{F}_{w}^{K}+\mathcal{F}_{w}^{K_{e}}\right)+\sum_{i=1}^{d}\left(\mathcal{F}_{z_{i}}^{K}+\mathcal{F}_{z_{i}}^{K_{e}}\right)\right\} d t \\
& -\sum_{e \in \Gamma_{h}} \int\left\{D_{\xi}^{K}\left(w_{K}, w_{K}\right)+D_{\xi}^{K_{e}}\left(w_{K_{e}}, w_{K_{e}}\right)\right\} \bar{\Psi}^{e} d t \\
& -\sum_{e \in \Gamma_{h}} \int\left\{\sum_{i=1}^{d}\left[D_{\xi}^{i, K}\left(z_{i, K}, z_{i, K}\right)+D_{\xi}^{i, K_{e}}\left(z_{i, K_{e}}, z_{i, K_{e}}\right)\right]\right\} \bar{\Psi}^{e} d t \\
& -\sum_{K \in \mathcal{T}_{h}} \int\left(J_{K} \bar{\Psi}_{t}^{K}+H_{K} \cdot \int_{K} \nabla_{x} \Psi d x\right) d t .
\end{aligned}
$$


Now for the $w$-terms in (4.14) we have, using the properties of the discrete fluxes,

$$
\begin{aligned}
-\frac{|e|}{|K|}\{ & \left.D_{\xi}^{K}\left(w_{K}, w_{K_{e}}\right)-D_{\xi}^{K}\left(w_{K}, w_{K}\right)\right\} \bar{\Psi}^{K} \\
& -\frac{|e|}{\left|K_{e}\right|}\left\{D_{\xi}^{K_{e}}\left(w_{K_{e}}, w_{K}\right)-D_{\xi}^{K_{e}}\left(w_{K_{e}}, w_{K_{e}}\right)\right\} \bar{\Psi}^{K_{e}} \\
& -\left\{D_{\xi}^{K}\left(w_{K}, w_{K}\right)+D_{\xi}^{K_{e}}\left(w_{K_{e}}, w_{K_{e}}\right)\right\} \bar{\Psi}^{e} \\
& +\left\{D_{\xi}^{K}\left(w_{K}, w_{K_{e}}\right)+D_{\xi}^{K_{e}}\left(w_{K_{e}}, w_{K}\right)\right\} \bar{\Psi}^{e} \\
= & \left\{D_{\xi}^{K}\left(w_{K}, w_{K_{e}}\right)-D_{\xi}^{K}\left(w_{K}, w_{K}\right)\right\}\left\{\bar{\Psi}^{e}-\frac{|e|}{|K|} \bar{\Psi}^{K}\right\} \\
& +\left\{D_{\xi}^{K_{e}}\left(w_{K_{e}}, w_{K}\right)-D_{\xi}^{K_{e}}\left(w_{K_{e}}, w_{K_{e}}\right)\right\}\left\{\bar{\Psi}^{e}-\frac{|e|}{\left|K_{e}\right|} \bar{\Psi}^{K_{e}}\right\} \\
\leq & C a_{e}\left|w_{K}-w_{K_{e}}\right|\left|\bar{\Psi}^{e}-\frac{|e|}{|K|} \bar{\Psi}^{K}\right|+C a_{e}\left|w_{K}-w_{K_{e}}\right|\left|\bar{\Psi}^{e}-\frac{|e|}{\left|K_{e}\right|} \bar{\Psi}^{K_{e}}\right| .
\end{aligned}
$$

A similar inequality holds true for the $z$-terms in (4.14). Hence, summing back to the elements $K$,

$$
\begin{aligned}
E \leq & \sum_{K \in \mathcal{T}_{h}} \sum_{e \in \partial K} \int\left\{a_{e}\left|w_{K}-w_{K_{e}}\right|+\sum_{i=1}^{d} b_{e}^{i}\left|z_{i, K}-z_{i, K_{e}}\right|\right\}\left|\bar{\Psi}^{e}-\frac{|e|}{|K|} \bar{\Psi}^{K}\right| d t \\
& -\sum_{K \in \mathcal{T}_{h}} \int\left(J_{K} \bar{\Psi}_{t}^{K}+H_{K} \cdot \int_{K} \nabla_{x} \Psi d x\right) d t .
\end{aligned}
$$

To adjust to the notation of Lemma 4.1 , let $\mathcal{S}_{h}=\left\{S^{K}\right\}, S^{K}=([0,+\infty) \times K)$, $K \in \mathcal{T}_{h}$ be a partition of $[0,+\infty) \times \mathbb{R}^{d}$. Then, for any $t>0,\left(S^{K}\right)_{t}=K$.

Further, we set

$$
\left.B_{H}\left(\nabla_{x} \Psi\right)\right|_{S^{K}}(x, t)=\frac{1}{|K|}|| e\left|\Psi(x, t)-\bar{\Psi}^{e}(t)\right| .
$$

Then, since $x \in K$, we have

$$
\begin{aligned}
\frac{1}{|K|}|| e\left|\Psi(x, t)-\bar{\Psi}^{e}(t)\right| & =\frac{1}{|K|}|| e\left|\Psi(x, t)-\int_{e} \Psi(S, t) d S\right| \\
& \leq \frac{1}{|K|} C h_{K}|e| \sup _{x^{\prime} \in K}\left|\nabla \Psi\left(x^{\prime}, t\right)\right| \leq C \sup _{x^{\prime} \in K}\left|\nabla \Psi\left(x^{\prime}, t\right)\right|,
\end{aligned}
$$

i.e., (4.4) is satisfied.

In view of (4.15), $U_{h}$ satisfies (4.2) with $\mathcal{S}_{h}=\left\{S^{K}\right\}, K \in \mathcal{T}_{h}$ as above, and

$$
\begin{aligned}
& \left.\alpha_{H}\right|_{S^{K}}=\left|H_{K}\right|,\left.\quad \alpha_{G}\right|_{S^{K}}=\left|J_{K}\right|, \\
& \left.\beta_{H}\right|_{S^{K}}=C \sum_{e \in \partial K}\left\{a_{e}\left|w_{K}-w_{K_{e}}\right|+\sum_{i=1}^{d} b_{e}^{i}\left|z_{i, K}-z_{i, K_{e}}\right|\right\} .
\end{aligned}
$$

Next, we will estimate the terms on the right-hand side of (4.5) in our case for $\nu=0, \Delta=\Delta^{\prime}$ and $u_{h}=U_{h}$. The only nonzero $E$-terms are $E^{H}, E^{G}$ and $\tilde{E}^{H}$. By 
(4.17), (4.8) and Lemma 3.3, we obtain for $R, T$ fixed,

$$
\begin{aligned}
E^{H} & \leq \frac{1}{\Delta}\left\{\iint_{0 \leq t \leq T}\left(\alpha_{H}(t, x)\right)^{2} d x d t\right\}^{1 / 2}\left\{\int_{0 \leq t \leq T}\left|B_{t}\right| d t\right\}^{1 / 2} \\
& \leq \frac{1}{\Delta}(R+M T)^{d / 2} T^{1 / 2} C \varepsilon^{1 / 2} .
\end{aligned}
$$

Similarly, (4.17), (4.6) and Lemma 3.4 imply

$$
\begin{aligned}
E^{G} & =\left(1+\frac{(1+M) T}{\Delta}\right) \sup _{0 \leq t \leq 2 T} \int_{B_{t}} \alpha_{G}(t, x) d x \\
& \leq(R+M T)^{d / 2} C_{\eta}\left(1+\frac{M T}{\Delta}\right) \varepsilon^{1 / 2-\eta / 2} .
\end{aligned}
$$

Finally, (4.17) and Lemma 3.3 yields

$$
\begin{aligned}
\tilde{E}^{H} & \leq \frac{1}{\Delta} C \int_{0 \leq t \leq T} \sum_{K \cap B_{t}^{\Delta}}|K| \sum_{e \in \partial K}\left\{a_{e}\left|w_{K}-w_{K_{e}}\right|+\sum_{i=1}^{d} b_{e}^{i}\left|z_{i, K}-z_{i, K_{e}}\right|\right\} \\
& \leq \frac{C h^{1 / 2}}{\Delta}(R+M T)^{d / 2} T^{1 / 2} .
\end{aligned}
$$

Using the above estimates in Lemma 4.1 we have, for $t \leq T$,

$$
\begin{aligned}
& \int_{|x|<R}\left|u_{h}(t, x)-u(t, x)\right| d x \\
& \quad \leq C\left(\Delta+\frac{(R+M T)^{d / 2} T h^{1 / 2}}{\Delta}\right)+\int_{B_{0}}\left|U_{h}(0, x)-u(0, x)\right| d x,
\end{aligned}
$$

and the proof of the theorem is complete by minimizing over $\Delta$.

\section{Fully DiscRete SCHEMES}

In this section we will briefly discuss the convergence of fully discrete finite volume schemes for (1.1-1.2) defined in Section 2. One can prove the following result:

Theorem 5.1. Let $u$ be the entropy solution of the conservation law (1.3) with initial data $u_{0} \in B V\left(\mathbb{R}^{d}\right) \cap L^{\infty}\left(\mathbb{R}^{d}\right)$, and let $\left\|u_{0}-\left(w_{0}^{\varepsilon}-\sum_{i=1}^{d} h_{i}\left(w_{0}^{\varepsilon}\right)\right)\right\|_{L^{1}} \leq C \varepsilon$. We denote $U_{h, \delta}=w_{h, \delta}-\sum_{i=1}^{d} h_{i}\left(w_{h, \delta}\right)$, where $\left(w_{h, \delta}, Z_{h, \delta}\right)$ is the solution of the finite volume scheme (2.7). In addition to (A.1-A.3) we assume that

$$
\left(-\frac{\partial G_{i}}{\partial z_{i}}-\sum_{j}\left|\frac{\partial G_{j}}{\partial w}\right|\right) \geq c_{1}>0 \quad \text { in } \mathcal{R}^{a, b} .
$$

Let $\varepsilon^{1-\eta} \leq C \delta$, where $1>\eta>0$ is any small number. If the $C F L$ condition $\frac{\delta|\partial K|}{|K|} \leq C_{0}$ holds, then for any time $t \leq T$, and $R>0$ there is a constant $C=$ $(R+M T)^{d / 4} T^{1 / 2} c(a, b)$ such that the following error estimate holds

$$
\left\|u(\cdot, t)-U_{h, \delta}(\cdot, t)\right\|_{L^{1}(B(0, R))} \leq C\left(h^{1 / 4}+\delta^{1 / 4}\right)+\left\|u(\cdot, 0)-U_{h, \delta}(\cdot, 0)\right\|_{L^{1}} .
$$

A detailed proof of Theorem 5.1 can be found in [KM]. It requires to prove the time discrete analogs of the estimates in Lemmata 3.1-3.4. In addition the 
following estimate is needed:

$$
\sum_{K \in \mathcal{T}_{h}}|K|\left(\left|w_{K}^{n+1}-w_{K}^{n}\right|+\sum_{i}\left|z_{i, K}^{n+1}-z_{i, K}^{n}\right|\right) \leq C \delta
$$

It should be emphasized that the additional assumption (5.1), which was used in the error estimates of [KT1 and [KKM] but not in Theorem 4.1 for the semidiscrete schemes, is now used only to prove

$$
\sum_{K \in \mathcal{T}_{h}}|K|\left(\left|w_{K}^{1}-w_{K}^{0}\right|+\sum_{i}\left|z_{i, K}^{1}-z_{i, K}^{0}\right|\right) \leq C \delta
$$

which in turn is used in the proof of

$$
\sum_{K \in \mathcal{T}_{h}}|K| \sum_{i}\left|G_{i}\left(w_{K}^{n}, z_{i, K}^{n}\right)\right|^{2} \leq C \delta .
$$

Therefore Theorem 5.1 still holds true if we replace assumption (5.1) by (5.2).

Note that most of the additional technical difficulties in the proofs of the time discrete stability estimates are focused on the time discrete analog of Lemma 4.3 and in the proof of (5.3) above.

\section{ACKNOWLEDGMENTS}

We would like to thank B. Cockburn, M. Katsoulakis, G. Kossioris, and A. Tzavaras for many valuable discussions. This work is partially supported by the GRSRT through PENED Program, No. 1747, and by the European Union TMR grant No. FMRXCT960033 (HCL).

\section{REFERENCES}

[BP] F. Bouchut and B. Perthame, Kruzhkov's estimates for scalar conservation laws revisited, Trans. Amer. Math. Soc. 350 (1998), 2847-2870. MR 98m:65156

[CLL] G.-Q. Chen, C. D. Levermore and T.-P. Liu, Hyperbolic conservation laws with stiff relaxation terms and entropy, Comm. Pure Appl. Math. 47 (1994), 789-830. MR 95h:35133

$[C] \quad$ B. Cockburn, On the continuity in $B V(\Omega)$ of the $L^{2}$-projection into finite element spaces, Math. Comp. 57 (1991), 551-561. MR 92a:65288

[CCL] B. Cockburn, F. Coquel and P. LeFloch, An error estimate for finite volume methods for conservation laws, Math. Comp. 63 (1994), 77-103. MR 95d:65078

[CGa] B. Cockburn and H. Gau, A posteriori error estimates for general numerical methods for scalar conservation laws, Mat. Apl. Comput. 14 (1995), 37-47. CMP 95:15

[CG1] B. Cockburn and P.-A. Gremaud, Error estimates for finite element methods for scalar conservation laws, SIAM J. Numer. Anal. 33 (1996), 522-554. MR 97e:65096

[CG2] B. Cockburn and P.-A. Gremaud, A priori error estimates for numerical methods for scalar conservation laws. Part I: The general approach, Math Comp 65 (1996), 533-573. MR 96g:65089

[CG3] B. Cockburn and P.-A. Gremaud, A priori error estimates for numerical methods for scalar scalar conservation laws. Part II: Flux-splitting monotone schemes on irregular Cartesian grids, Math Comp 66 (1997), 547-572. MR 97m:65173

[CGY] B. Cockburn, P.-A. Gremaud and X. Yang, A priori error estimates for numerical methods for scalar scalar conservation laws. Part III: Flux-splitting monotone finite volume schemes, SIAM J. Numer. Anal. 35 (1998), 1775-1803. MR 99g:65097

[CM] M. Crandall and A. Majda, Monotone difference approximations for scalar conservation laws, Math. Comp. 34 (1980), 1-21. MR 81b:65079

[EG] R. Eymard, T. Gallouët, M. Ghilani and R. Herbin, Error estimates for the approximate solutions of a nonlinear hyperbolic equation given by the finite volume schemes, IMA J. Numer. Anal. 18 (1998), 563-594. CMP 99:11 
[GM] L. Gosse and Ch. Makridakis, A posteriori error estimates for numerical approximations to scalar conservation laws: Schemes satisfying strong and weak entropy inequalities. FORTH-IACM Technical Report 98.4 (1998).

[JX] S. Jin and Z. Xin, The relaxing schemes for systems of conservation laws in arbitrary space dimensions, Comm. Pure Appl. Math. 48 (1995), 235-277. MR 96c:65134

$[\mathrm{KM}] \quad$ Th. Katsaounis and Ch. Makridakis, Finite volume relaxation schemes for multidimensional conservation laws,, Preprint 97-12, Dept of Math., Univ. of Crete (1997).

[KZ] Th. Katsaounis and G. Zouraris, Numerical evaluation of relaxation schemes for multidimensional conservation laws, (To appear).

[KKM] M.A. Katsoulakis, G. Kossioris and Ch. Makridakis, Convergence and error estimates of relaxation schemes for multidimensional conservation laws, Comm. Partial Differential Equations 24 (1999), 395-424. CMP 99:11

[KT1] M.A. Katsoulakis and A.E. Tzavaras, Contractive relaxation systems and the scalar multidimensional conservation law, Comm. Partial Differential Equations 22 (1997), 195233. MR 97m:35168

[KR] D. Kröner and M. Rokyta, Convergence of upwind finite volume methods for scalar conservation laws in two dimensions, SIAM J. Numer. Anal. 31 (1994), 324-343. MR 95e:65085

[Kr] S.N. Kruzhkov, First order quasilinear equations with several independent variables, Math. USSR Sbornik 10 (1970), 217-243.

[Kz] N.N. Kuznetzov, Accuracy of some approximate methods for computing the weak solutions of a first-order quasi-linear equation, USSR Comp. Math. and Math. Phys. 16 (1976), 105-119.

[N1] R. Natalini, Convergence to equilibrium for the relaxation approximations of conservation laws, Comm. Pure Appl. Math. 8 (1996), 795-823. MR 97f:35131

[N2] R. Natalini, A discrete kinetic approximation of entropy solutions to multidimensional scalar conservation laws, J. Differential Equations 148 (1998), 292-317. MR 99e:35139

[R] C. Rohde, Upwind finite volume schemes for weakly coupled hyperbolic systems of conservation laws in 2D, Numer. Math. 81 (1998), 85-123. MR 99j:65174

[S] R. Sanders, On convergence of monotone finite difference schemes with variable spatial differencing, Math. Comp. 40 (1983), 91-106. MR 84a:65074

[ScTW] H. J. Schroll, A. Tveito and R. Winther, An $L^{1}$ error bound for a semi-implicit difference scheme applied to a stiff system of conservation laws, SIAM J. Numer. Anal. 34 (1997), 1152-1166. MR 98g:65078

[ShTW] W. Shen, A. Tveito and R. Winther, A system of conservation laws including a stiff term; the 2D case, BIT 36 (1996), 786-813. MR 98c:65157

[V] J.-P. Vila, Convergence and error estimates in finite volume schemes for general multidimensional scalar conservation laws. I. Explicit monotone schemes, RAIRO Modél. Math. Anal. Numér. 28 (1994), 267-295. MR 96d:65150

Ecole Normale Supérieure, Département de Mathématique et d'Informatique, 45 rue D'Ulm, 75230 Paris Cedex 05, France

E-mail address: Theodoros.Katsaounis@ens.fr

E-mail address: thodoros@math.uch.gr

Department of Mathematics, University of Crete, 71409 Heraklion, Crete, and Institute of Applied and Computational Mathematics, FORTH, 71110 Heraklion, Crete, GREECE

E-mail address: makr@math.uch.gr 